\title{
CONSIDERACIONES SOBRE ALGUNOS ASPECTOS Y PROBLEMAS DEL ANÁLISIS DEL DISCURSO
}

LAura Alba Juez UNED. Madrid

\section{RESUMEN}

En este artículo se consideran algunos aspectos y problemas del análisis del discurso tales como la obtención de datos y la ética para su recogida, el enfoque a seguir, las unidades a elegir, el contexto, problemas epistemológicos tales como la relación entre el discurso y el mundo real o la verdad objetiva de los resultados, etc. Se discuten estos aspectos con objeto de dar lugar a la reflexión sobre el tema, considerando que son problemas, aún sin resolver, que de alguna manera limitan el alcance de esta disciplina como métodos de análisis, y que si los investigadores de este campo los conocen y son conscientes de ellos, tendrán más capacidad para resolverlos.

\section{INTRODUCCIÓN}

A pesar del momento pujante por el que atraviesan los estudios discursivos en la actualidad, no podemos dejar de analizar y reconocer que este enfoque lingüístico tiene aún ciertos problemas sin resolver que de alguna manera limitan su alcance como método de análisis. Asimismo, existen aspectos dentro del enfoque 
que todavía deben ser mejorados y estudiados con mayor profundidad, dado que se trata de una corriente lingüística relativamente nueva de pensamiento y análisis. Entre estos aspectos y problemas consideraremos aquí los siguientes:

a) Los datos: obtención y ética para su recopilación.

b) El tipo de discurso y la(s) unidad(es) a elegir

c) El enfoque a seguir

d) El contexto: ¿Qué aspectos podemos considerar relevantes y cuáles no?

e) Problemas epistemológicos: La relación entre el discurso y el mundo real, la verdad objetiva de los resultados y su generalización.

f) El discurso objeto de estudio: ¿Tema o recurso? ¿Proceso o contenido?

g) La identidad del investigador durante la recogida y análisis de los datos.

El objetivo principal de este trabajo es simplemente presentar estos aspectos sin resolver para dar lugar a la reflexión y discusión del tema, dado que es de esperar que si los investigadores de un campo conocen los problemas inherentes a su disciplina, tratarán de poner su empeño en resolverlos más rápidamente que si no los conocen. Como investigadora dentro del campo del análisis del discurso, me interesa entonces poner todos estos aspectos sobre el tapete, sin pretender por ello encontrar una respuesta totalmente clarificadora de los interrogantes.

Veamos ahora, cada uno de estos aspectos en particular.

\section{Los Datos: OBTENCIÓN Y ÉTICA PARA SU RECOPILACIÓN}

Uno de los primeros problemas que se nos plantean al enfrentarnos al análisis del discurso es cómo recoger los datos. Existen diferentes posibilidades, como pueden ser la grabación y posterior trascripción del fragmento del discurso en cuestión, el uso de textos que están ya disponibles en el ordenador o en la red de internet, etc. El conjunto de datos con los que trabajamos, sea compilado como textos escritos o sea una trascripción del discurso hablado, es lo que en linguística se conoce con el nombre de corpus. La lingüística de corpus es una rama de la lingüística que precisamente se ocupa de los principios y la práctica de usar corpus para el estudio del lenguaje. Existen diferentes tipos de corpus, siendo los corpus computarizados los más usados actualmente, debido a la gran cantidad de datos e información que se puede obtener de ellos. Dependiendo de los objetivos de estudio, es útil también usar programas de trata- 
miento de corpus como por ejemplo los llamados «Concordance» (como el Wordsmith, por ejemplo), que permiten, entre otras cosas, ubicar todos los contextos en los que aparece una determinada palabra o expresión. Estos últimos se usan profusamente en áreas de estudio como la lexicografía, y también pueden ser muy útiles dentro de un enfoque discursivo variacional.

La forma en que se transcriben los datos representa ya de por sí un problema, pues cada autor usa diferentes convenciones y en ningún caso se puede decir que se trate de una trascripción perfecta, neutral y totalmente fiel. Algunos autores han intentado reflejar no sólo los rasgos sintácticos sino también los prosódicos en la trascripción, como es el caso de Svartvik \& Quirk (1980) en el famoso London Lund Corpus of English Conversation. En muchos casos se trata también de dar los datos contextuales sobre los interlocutores, el lugar y tiempo de la interacción, etc., para ayudar al análisis pragmático.

Como señalan Jaworski \& Coupland (1999: 36), el análisis del discurso es primariamente una orientación cualitativa al entendimiento del lenguaje y la sociedad, y consecuentemente ha heredado tanto los puntos fuertes como los débiles de la investigación cualitativa. Un punto débil es el problema de justificar la selección de los materiales que han de constituir los datos de la investigación, es decir que es generalmente difícil justificar por qué hemos elegido un determinado fragmento de conversación o un determinado texto escrito y no otro, o por qué se presta atención a algunas de sus características y no a otras.

Otro de los problemas en relación con los datos es el de la manera en que son recopilados. Debe existir una cierta ética de la investigación, dado que no se puede ignorar que los participantes del discurso que estamos estudiando son seres humanos y el investigador no debe imponerse sobre los derechos de éstos. En general, y como observan Wetherell et al. (2001a), el investigador tiene un cierto poder sobre los sujetos participantes (dado que tiene más información sobre el proyecto), pero debe de tener mucho cuidado de no abusar de su poder. Como primera medida, se debe garantizar el anonimato de los participantes (aunque, como hemos visto, en algunos casos esto es imposible, ya que si, por ejemplo se hace un análisis crítico del discurso de un determinado político, la intención es generalmente de denuncia y se quiere que se sepa quién es el hablante). Por otro lado, otro requerimiento de importancia es que el investigador obtenga el consentimiento de los sujetos de estudio para el proyecto. Además, se deben observar los derechos legales de los participantes, lo cual puede resultar complejo, especialmente si se trabaja con documentos legales o médicos o con participantes menores de edad (Wetherell et al., $2001 \mathrm{a})$. 


\section{EL TIPO DE DISCURSO Y LA(S) UNIDAD(ES) A ELEGIR}

Como cualquier hablante puede intuir, existen distintos tipos de discurso, $y$, si como investigadores emprendemos la tarea del análisis de un determinado problema, una de las primeras consideraciones será también el decidir qué tipo de discurso vamos a estudiar. No es tarea fácil hacer una relación entre las formas lingüísticas y el tipo de discurso, ya que con frecuencia se usan las mismas formas en distintos tipos de discurso. Existen otras muchas variables a tener en cuenta a la hora de clasificar el discurso, y según consideremos unas u otras podemos clasificarlo de manera diferente. Podemos hablar del discurso legal, el discurso médico o el periodístico, pero aún dentro de clases como éstas podemos hacer otras subdivisiones.

Es también necesario decidir con qué unidades vamos a trabajar. Como sabemos, las distintas escuelas trabajan con distintas unidades, como los actos de habla, los turnos o los pares adyacentes, pero hasta el momento no existe un consenso en cuanto a cuál sea la unidad discursiva por excelencia, y, por ello, es por lo que se utilizan criterios muy dispares. Lakoff (1990) y 1998 (comunicación personal) explica que el turno o el acto de habla, por ejemplo, serían unidades «menores» de análisis y presenta una taxonomía con diferentes tipos de discurso, que, a su vez, podrían considerarse unidades «mayores» de análisis, entre las cuales se encuentran las siguientes:

- Formall informal

- Recíprocol no recíproco

- Espontaneol no espontaneo

- Interacción personal /conversación telefónica

- Públicol privado

- Discurso orientado hacia un propósito o tarea particular (por ejemplo, el discurso psicoterapéutico)

- Discurso literario (incluye todos los modos de discurso lingǘístico escrito)

- Discurso memorable (cuya intención es que perdure y se grabe y conserve para el futuro).

- Discurso enfático (en el que podemos ver lo que cada interlocutor siente en el momento. Ej.: diálogo o conversación normal)

- Discurso monológico (en el cual uno de los participantes tiende a acaparar el discurso )

- Discurso orientado hacia la verdad (diseñado con el propósito de encontrar la verdad de los hechos. Ej.: discurso legal) versus discurso de ficción (Searle, 1979) 
- Discurso hablado/discurso visual (gestos, movimientos, etc.) (Fairclough, 1989)

- Discurso diádico/triádico/grupo (Varios hablantes pueden participar. La escritura no es generalmente diádica, pero las cartas o comunicación por correspondencia sí lo son) (Simmel, 1950)

Es muy interesante también la caracterización que E. Bernárdez (1995, capítulo XII) hace sobre los tipos de texto como configuraciones prototipicas de estrategias. Este autor, al igual que otros, nos hace ver que el problema de la tipología del texto y del discurso es muy complejo, y si bien de una manera acientífica podemos distinguir entre un tipo y otro, en realidad no tenemos fundamentos totalmente válidos para hablar de distinciones absolutas entre ellos. Por ello me parece oportuno citar una vez más a Robin Lakoff en su apreciación de que debemos hablar más de «un continuo» de tipos discursivos que de categorías completamente distintas unas de otras (Lakoff, comunicación personal, 1998).

\section{El ENFOQue a SEguir}

Como ya hemos visto, existen diferentes enfoques o aproximaciones al análisis del discurso, y esto también puede convertirse en un problema a la hora de comenzar un proyecto. ¿Por qué elegir una corriente y no otra? ¿Cómo estamos seguros de que el enfoque que hemos elegido es el apropiado? Wetherell et al. (2001b: 380) explican que el tipo de investigación que se favorece para un determinado proyecto involucra necesariamente un equilibrio entre $e l$ tipo de datos que el investigador quiere recoger, el tema, la disciplina académica en la que se está trabajando y la tradición discursiva que se adecua más al tipo de problema a tratar.

La demarcación de los límites está más definida entre algunos enfoques que entre otros. Por ejemplo, las diferencias entre el análisis de la conversación y el análisis de la escuela post-estructuralista (Focault, 1980) son obvias '. Otras tradiciones son más de tipo híbrido y se adaptan a distintos objetivos.

Por otro lado, se debe considerar si el investigador está interesado en tomar una posición política y comprometerse con una causa o si, por el contrario, considera que debe ser un observador neutro. Es evidente que, en el primer

' Es conocido el hecho de que Focault no estaba interesado en el microanálisis o la interacción social. El análisis de la conversación, por el contrario, sí lo está, y ofrece una perspectiva diferente del orden y la acción social. 
caso, el enfoque a adoptar debería ser el análisis del discurso crítico, el cual tiene como objetivo la denuncia de la injusticia social y política a través del estudio del discurso, y además exige un compromiso político y social por parte del investigador. Un representante claro de este enfoque es Teun Van Dijk, cuyos trabajos de investigación en el campo del Análisis del discurso apuntan a denunciar ciertas injusticias políticas y sociales (como el racismo, por ejemplo) por medio del estudio del discurso de ciertos políticos o gente con poder (Van Dijk, 2001). En este sentido se podría decir que el análisis de la conversación es totalmente opuesto al análisis del discurso crítico, pues sus representantes, entre los cuales se encuentra Schegloff, argumentan que el mezclar la investigación con la política implica no solamente hacer mala investigación, sino mala política (Wetherell et al. $200 \mathrm{lb}$ ).

\section{El CONTEXTO: ¿QUÉ ASPECTOS PODEMOS CONSIDERAR RELEVANTES Y CUÁLES NO?}

Otra de las preguntas que se hace el investigador al enfrentarse con el análisis del discurso tiene que ver con la cantidad de información del trasfondo contextual que es necesario tener en cuenta. Una vez más la decisión dependería del enfoque adoptado: el análisis de la conversación, por ejemplo, sostiene que el único contexto necesario es el de las actividades que tienen alguna relevancia para los participantes según su discurso lo revele, es decir, sólo es de las actividades o cosas que evidentemente tengan alguna consecuencia para la conversación en particular. Muchos investigadores del discurso, sin embargo, favorecen la visión amplia del contexto, es decir, la visión que considera la conversación dentro de un contexto social, histórico, político y cultural, la cual tiene que ver con la visión post-estructuralista (Focault). En realidad, dentro de la posición post-estructuralista es difícil distinguir entre lo discursivo y lo extra-discursivo, ya que ambos forman parte de un todo indivisible.

A modo de resumen, y siguiendo a Whetherell et al. (2001b), podemos decir que lo que se utilice como datos contextuales en un determinado estudio depende en parte del objeto de estudio, pero principalmente depende del enfoque adoptado, es decir de la perspectiva que se tenga de la interrelación entre lo discursivo y lo social.

A mi juicio, ninguna perspectiva es mejor que otra, pero sí creo que al emprender la tarea de un análisis discursivo, el contexto es de capital importancia y, por lo tanto, cuanta más información contextual se tenga, mejor preparados estaremos para llevar la investigación a buen fin. 
6. Problemas EPISTEMológicos: La RELACión ENTRE El disCuRSO Y El MUNDO REAL, LA VERDAD OBJETIVA DE LOS RESULTADOS Y SU GENERALIZACIÓN

La mayoría de los enfoques discursivos cuestionan el supuesto simplista de que el lenguaje es neutral y transparente y de que refleja el mundo real. Se considera que el discurso desempeña actividades y funciones muy complejas que van más allá de la función referencial del lenguaje, y aún más, que la realidad está construida en forma discursiva. Esta última posición es más extrema y evidentemente refleja la visión post-estructuralista de Focault y sus seguidores.

La posición contraria a la del post-estructuralismo es la que sostiene la existencia de una realidad subyacente separada del discurso o de la forma en la que hablamos de ella. Esta posición es parte de lo que se ha llamado nuevo realismo o realismo crítico (Bhaskar, 1978). Algunos analistas del discurso, como Fairclough (1995, 2001), son realistas y rechazan el relativismo, dado que éste llevaría al convencimiento de que no hay ninguna verdad absoluta, lo cual, a su juicio, le quitaría sentido a la investigación, pues si no hubiera ninguna verdad que descubrir, no valdría la pena esforzarse en investigar problema alguno. Los relativistas, por el contrario, encuentran que el sentido de la investigación no está en encontrar la verdad absoluta, sino en estudiar la forma en que cada uno construye su realidad a través del discurso.

El enigma de la realidad objetiva es un problema filosófico que se ha venido discutiendo en el ámbito de muchas disciplinas desde tiempo inmemorial, y, por lo tanto, sería justamente poco realista pensar que aquí podemos encontrar una respuesta al dilema de cómo considerar la realidad desde nuestro análisis. Considero, sin embargo, necesario, al menos, mencionar que existen polémicas en torno al tema para poder enfrentarnos a la tarea de análisis con un conocimiento más profundo de los problemas implícitos.

Ahora bien, el planteamiento de la relación entre el discurso y la realidad nos lleva a otra pregunta: ¿Cuán objetivos y cercanos a la verdad/realidad son nuestros resultados? Muchos investigadores parten de la base de que su trabajo es válido si cumple con los criterios de la comunidad académica en la que se desenvuelven, pero el concepto de validez es muy complejo, y no es contemplado por todos los investigadores del discurso de la misma forma (Wetherell et al, 2001). Por ejemplo, los analistas de la conversación, tienen un interés limitado en los debates filosóficos, y su posición tiende a ser una de gran escepticismo frente a las teorías abstractas. El análisis de la conversación se inclina más bien por una posición empírica, que defiende la posición de mirar y analizar la conversación para ver lo que se encuentra y lo que se puede aprender de ella. Se asume que el estudio exhaustivo de la conversación reve- 
la regularidades empíricas sobre realidades como el uso de los turnos, las cuales pueden ser descritas objetivamente.

Otros enfoques, por el contrario, critican el estilo excesivamente empírico del análisis conversacional, argumentando que la identificación de ciertos patrones (como los turnos o los pares adyacentes) dependen de supuestos preconcebidos, y, por tanto, no hay ningún tipo de conocimiento que pueda ser totalmente objetivo; es mejor tratar de interpretar de una manera reflexiva los hechos a estudiar. La llamada «crisis de la representación» es un problema que pone de manifiesto la imposibilidad del investigador para ofrecer un conocimiento objetivo de la realidad del mundo, ya que siempre debe hacerlo a través de su punto de vista subjetivo y está atrapado por él, de manera que los resultados de su investigación nos dicen más de su visión del mundo que de la del mundo real.

Un tema relacionado con lo anterior es el de la posibilidad de generalizar los resultados de la investigación. Una vez más, la respuesta está en las premisas o supuestos de los que parta el investigador. Wetherell et al. (2001b) señalan que si el análisis se centra en el lenguaje como un sistema en sí mismo, la tendencia es a proclamar la generalización y universalización de los resultados. Si el análisis se centra en la interacción es, tal vez, aún posible generalizar, pero el fundamento para hacerlo será diferente y más limitado a los fenómenos particulares dentro de la interacción. Como contrapartida de la generalización, existen estudios cuyo objetivo es apuntar a un fenómeno discursivo que tiene un significado particular en sí mismo, en cuyo caso la generalización no tiene sentido. El punto aquí no es destacar que un determinado rasgo o fenómeno es recurrente, sino que es significativo y persistente dentro de, por ejemplo, una sociedad particular.

Finalmente, es también interesante que nos cuestionemos quién tiene la verdad en cuanto a la determinación e interpretación de los datos discursivos. ¿Se debe dar más peso a la interpretación del analista, a su «autoridad intelectual» $o$ a la «verdad manifiesta» en el discurso por los participantes de la interacción? Es claro que un enfoque como el análisis de la conversación da más importancia a los participantes que al investigador, mientras que otros enfoques (como los formalistas) dan más credibilidad a la interpretación del investigador.

Existen trabajos que intentan hallar una fundamentación científica (y por lo tanto intentan hallar la verdad objetiva) para la lingüística textual y otras disciplinas lingüísticas, entre los cuales se encuentra el estudio de Enrique Bernárdez (1995) sobre la teoría y epistemología del texto, en el cual esta fundamentación científica se basa en la Caología o Teoría de Catástrofes.

Los problemas epistemológicos requieren un estudio y consideración mucho más profundos de los que se les puede dar en un artículo como éste; no obs- 
tante, como se ha dicho más arriba, he considerado necesario e importante mencionarlos ya que, a mi entender, deben de ser tenidos en cuenta por cualquier investigador con una perspectiva analítica rigurosa.

\section{El Discurso Objeto de Estudio: ¿TEMA O ReCURSo? ¿PROCESO O CONTENIDO?}

Otro de los debates en el análisis del discurso se refiere al estudio del lenguaje como tópico o como recurso, es decir, si el interés es el estudio del lenguaje en sí mismo o si se usa el lenguaje como un recurso para estudiar algo más. En general, dentro del análisis del discurso, los investigadores se aproximan al lenguaje como un tópico en sí mismo, pero la disyuntiva tópico/recurso siempre sale a la superficie de alguna manera, especialmente en enfoques como el análisis crítico, cuyo objetivo es, como ya hemos dicho, la denuncia de injusticias sociales y políticas. En este caso, es claro que, si bien se toma inicialmente al discurso como un tópico, los resultados de la investigación lo convierten en un recurso, pues el fin último del investigador tiene más que ver con su compromiso social y político que con su condición de lingüista. De cualquier manera, la frontera entre estos dos conceptos no está tan claramente delimitada ni aún en los enfoques más formalistas.

Muchos investigadores se preguntan también si deben dar más prioridad al proceso que al contenido, o viceversa. Si damos prioridad al proceso, estaremos probablemente interesados en la interacción en curso, probablemente hablada, así como en la manera en que los hablantes se comunican y ejecutan acciones a través del habla. Si, por el contrario, damos prioridad al contenido, el uso del lenguaje se analiza como un todo, como si fuera un producto acabado. Esta distinción entre proceso en curso y contenido/producto es muy importante dentro de enfoques como la etnometodología y el análisis de la conversación, en los cuales se cree que dentro de una interacción, los hablantes van creando el significado conjuntamente a medida que avanza la conversación, y, por lo tanto, prevalece la visión del lenguaje como proceso.

8. LA IDENTIDAD DEL INVESTIGADOR DURANTE LA RECOGIDA Y ANÁLISIS DE LOS DATOS

Un aspecto que también se considera de importancia es el papel que juega el investigador dentro del análisis, y hasta qué punto puede separarse de su investigación. Wetherell et al. (2001a) analizan el concepto de reflexividad, que 
sugiere que la separación es imposible, lo cual conlleva implicaciones sobre la identidad del investigador de cara a la recopilación y análisis de los datos.

Efectivamente, muchos investigadores argumentan que el distanciamiento es imposible, y en consecuencia siempre se tiene que tener en cuenta la influencia que el investigador ha ejercido sobre los resultados de su estudio, lo cual implica tener en cuenta hasta qué punto su identidad es relevante para la investigación, especialmente en aquellos estudios que consideran la entrevista como un método de recopilación de datos. Por otro lado, es evidente que la identidad del investigador influye en áreas como la selección del tema de estudio, el cual depende siempre en gran medida de sus intereses personales.

En muchos casos, la identidad del investigador puede llegar a ser problemática y controvertida, hasta el punto de tener que abandonar la investigación y reconocer que no es la persona idónea para un determinado proyecto (Wetherell et al., 2001a).

\section{RESUMEN y CONCLUSIONES}

En este trabajo se ha intentado señalar algunos de los problemas implícitos en la investigación de corte discursivo y reflexionar sobre ellos. El planteamiento de estos problemas nos hace ver que no existe una panacea para su solución ni un enfoque linguístico que pueda controlarlos a todos.

Hemos visto que el manejo de los datos es uno de los primeros problemas que se nos presenta al intentar emprender un análisis, desde su recopilación, pasando por la forma en que son transcritos hasta la ética de su recogida. En todo momento debemos de hacer elecciones, que a mi juicio deberán de ser lo más objetivas posible, tratando también de respetar a los sujetos de estudio en caso de que los hubiera. Sin embargo, es difícil desprender al investigador de los datos y sujetos que (generalmente) él mismo seleccionó para su trabajo, por lo que pienso que es imposible la objetividad total en el análisis del discurso. El investigador generalmente estudia lo que le interesa dentro de los discursos y contextos que él considera más apropiados para sus objetivos, aún cuando siga las líneas metodológicas de una escuela determinada, con lo cual la variable de su intervención no puede estar totalmente controlada. Este hecho, sin embargo, no le quita, a mi juicio, validez a dichos estudios. Sí los hace, tal vez, más relativos a determinados contextos y bajo ciertas condiciones, siendo generalmente difícil proclamar su universalidad.

En lo relativo a la disyuntiva frente a qué enfoque y qué tipo de discurso o unidades debemos elegir para el análisis, todas las escuelas pueden considerarse como posibles opciones, dependiendo del objeto y condiciones de 
estudio en cada caso en particular. En ciertos casos puede ser de gran utilidad contrastar unos enfoques con otros con el objeto de observar y describir más claramente la naturaleza del fenómeno en cuestión. Prácticamente se puede decir que cada investigador tiene su propia metodología y enfoque, aunque, paradójicamente, las diferencias entre unos enfoques y otros no son a veces tan nítidas. Hay mucha superposición entre las distintas corrientes del análisis del discurso, pero también hay muchas diferencias entre un investigador y otro. No existe, hasta el momento, una unidad que pueda considerarse como la única o la mejor para el análisis. Generalmente las distintas escuelas trabajan con diferentes unidades; así un analista de la conversación trabaja con turnos y pares adyacentes, mientras que un investigador de corte eminentemente pragmático trabajará con actos de habla o presuposiciones, por ejemplo. Nuevamente podemos decir que los fines de la investigación determinarán la unidad o unidades de trabajo así como el tipo de discurso del cual sacar los datos.

También hemos visto que diferentes escuelas ven al contexto de distinta manera. Como se ha dicho antes, el tratamiento de los datos contextuales depende generalmente de la perspectiva que se tenga de la interrelación entre lo discursivo y lo social. La posición adoptada aquí favorece el análisis de la mayor cantidad posible de datos del contexto, dado que, en consonancia con la esencia de la visión discursiva del lenguaje, texto y contexto son inseparables.

Otros problemas aquí tratados han sido los epistemológicos en relación con la «verdad objetiva» de los datos, sus resultados y su generalización. Como ya se ha señalado, no se pretende dar respuesta a semejante disyuntiva filosófica, pero sí se intenta presentarla como tema de discusión y reflexión, a fin de ver cómo podemos conjugar posiciones extremas como la del post-estructuralismo, que, como ya sabemos sostiene que la realidad está construida de forma discursiva, y la del realismo crítico, que sostiene la existencia de una realidad subyacente y separada del discurso. En cuanto a los resultados, sería interesante también ver si podemos encontrar una posición intermedia entre la empírica, que asume que el estudio exhaustivo del discurso revela regularidades que pueden ser descritas objetivamente (como, por ejemplo, la del análisis de la conversación) y otros enfoques que, por el contrario, argumentan que no hay ningún tipo de conocimiento que pueda ser totalmente objetivo.

El hecho de señalar que el alcance de los estudios discursivos está limitado, en cierta medida, por los problemas aquí señalados no pretende, de ninguna manera, ir en detrimento de dichos estudios. Muy al contrario, la intención es arrojar luz sobre ciertos aspectos problemáticos (que, por otra parte, en su mayoría no son exclusivos del análisis del discurso sino también de otros mu- 
chos campos de investigación), con la esperanza de que cada vez seamos más capaces de producir estudios serios, coherentes y de calidad. Es mi opinión y la de otros muchos investigadores, que la visión discursiva del lenguaje nos ha abierto la mente y las puertas a un conocimiento más profundo y amplio del fenómeno humano del lenguaje.

\section{REFERENCIAS}

BERNÁRDEZ, E. (1995): Teoría y Epistemología del Texto. Madrid: Cátedra.

BHASKAR, R. (1978): A Realist Theory of Science. London: Harvester Press.

FAIRCLOUgh, N. (1989); «Discourse as social practice». En : Language and Power, pp. 17-42. London: Longman.

FouCAult, M. (1980): Power/Knowledge: Selected Interviews and Other Writings 1972-1977, translated by C. Gordon, L. MarshaLl, J. MEPHAM, and K. SOPER, and edited by C. Gordon. New York: Pantheon Books.

Fowler, R., Hodge, B. Dress, G. \& T. TRew (1979): Language and Control. London: Routledge and Kegan Paul.

JAWORSKI, A. \& N. Coupland (eds.) (1999): The Discourse Reader. London and New York: Routledge.

Lakoff, R. (1990): Talking Power: The Politics of Language. New York: Basic Books. Simmel, G. (1950): «Sociability», Part 1/III and «The expansion of the dyad», Part 2/III \& IV («The triad»), in K. Wolff (ed.) The Sociology of Georg Simmel. New York: Free Press.

SVARTVIK, J. \& QUIRK, R. (eds.) (1980): A Corpus of English Conversation. CWK GLEERUP LUND.

VAN Dijk, T. (2001): «Critical Discourse Analysis». D. Schiffrin, D. Tannen, \& H. Hamilton (eds.), The Handbook of Discourse Analysis, pp 352-371. Oxford: Blackwell.

Wetherell, M.; TAYLoR, S. \& YATES, S.J. (2001a): Discourse as data. London: Sage Publications.

- (2001b). Discourse Theory and Practice: a Reader. London: Sage Publications. 